\title{
Application of Support Vector Machines for Estimating Wall Parameters in Through-Wall Radar Imaging
}

\author{
Hua-Mei Zhang, ${ }^{1}$ Ye-Rong Zhang, ${ }^{1}$ Fang-Fang Wang, ${ }^{1}$ and Jun-Lin An ${ }^{2}$ \\ ${ }^{1}$ School of Electronic Science and Engineering, Nanjing University of Posts and Telecommunications, Nanjing 210003, China \\ ${ }^{2}$ Institute of Atmospheric Physics, Nanjing University of Information Science \& Technology, Nanjing 210044, China \\ Correspondence should be addressed to Hua-Mei Zhang; zhanghm@njupt.edu.cn
}

Received 27 November 2014; Accepted 25 April 2015

Academic Editor: Jaume Anguera

Copyright (c) 2015 Hua-Mei Zhang et al. This is an open access article distributed under the Creative Commons Attribution License, which permits unrestricted use, distribution, and reproduction in any medium, provided the original work is properly cited.

\begin{abstract}
In through-wall radar imaging (TWRI), ambiguities in wall characteristics including the thickness and the relative permittivity will distort the image and shift the imaged target position. To quickly and accurately estimate the wall parameters, an approach based on a support vector machine (SVM) is proposed. In TWRI problem, the nonlinearity is embodied in the relationship between backscatter data and the wall parameters, which can be obtained through the SVM training process. Measurement results reveal that once the training phase is completed, the technique only needs no more than one second to estimate wall parameters with acceptable errors. Then through-wall images are reconstructed using a back-projection (BP) algorithm by a finite-difference time-domain (FDTD) simulation. Noiseless and noisy measurements are discussed; the simulation results demonstrate that noisy contamination has little influence on the imaging quality. Furthermore, the feasibility and the validity are tested by a more realistic situation. The results show that high-quality and focused images are obtained regardless of the errors in the wall parameter estimates.
\end{abstract}

\section{Introduction}

Though-wall radar imaging (TWRI) is a form of nondestructive detection that has applications in civil engineering and security. In TWRI, if the wall parameters such as the wall thickness and the relative permittivity are known in advance, the changes in the speed and the amplitude attenuation of the electromagnetic waves that pass through the wall can be calculated accurately. Moreover, the refraction point of the electromagnetic waves can be determined precisely. Hence, targets behind walls can be easily detected and located. There have been many studies on TWRI that have created highquality images with known wall parameters [1-3]. However, wall parameters are generally not known a priori. If the wall parameters are not properly considered, the TWRI images will be fuzzy or distorted and the positions of the targets in the image will be incorrect.

Walls are commonly constructed with brick, wood, glass, plaster, or concrete block, and the propagation of EM waves in these materials differs. Efforts have been made to understand the effects of various common building materials. In addition, efforts have been made to image or locate the target through building walls with unknown characteristics [4-7]. Although these methods can provide high-quality, that is, focused, images, certain algorithms rely on knowledge of the wall parameters such as the dielectric constant, the wall thickness, and the conductivity.

In recent years, various studies have attempted to estimate the values of wall parameters. At several different transceiverreceiver separations various time-delay-only measurements were proposed for a wall parameter estimation. The estimated wall parameters can be utilized for improving the accuracy of real-time locating and tracking of moving humans $[8,9]$. Through setting various targets located at various distances on the other side of the wall, the frequency domain data was converted to time domain and then mapped with spatial domain to estimate the wall parameters. But this technique can only be experimented in a known environment [10]. Multichannel radar antennas were used to accurately and simultaneously estimate both the wall parameters and the target position [11]. A linear trajectory was fitted to a set of target images assuming various wall thicknesses via the 
Radon transform for each assumed dielectric constant [12]. The intersection of the trajectories corresponding to the various dielectric constants was taken as the estimated target position, which was then used to obtain estimates of the wall thickness and the dielectric constant. All above methods require the use of two or more antenna arrays or at least two measurements. Measurement methods are effective in a lab, but their usefulness is limited in practice. Other methods construct a cost function between wall parameters and a computer index, such as the sharpness of the target images and the entropy $[13,14]$. These methods attempt to minimize the cost function and can produce satisfactory images, but they impose a significant computational burden. The estimation of wall parameters was approached as a least squares problem based on a forward model of electromagnetic wave propagation in a homogeneous wall [15]. Two filter-based approaches were developed and can provide quick and precise estimates of wall parameters, but they do not work well in low-SNR conditions.

In this study, we use a novel approach to obtain quick and precise estimates of wall parameters. The approach is based on SVMs. The SVM, which was introduced by Vapnik, is a statistical learning method. The SVM is based on the VC dimension and structural risk minimization. By solving a convex quadratic programming problem, SVMs can find the global optimal solution rather than local minima and avoid overfitting. SVMs have a strong theoretical foundation and good generalization performance. The algorithm is based on limited sample information and can provide real-time results. Originally, SVMs were developed for solving binary classification problems. Recently, SVMs have been used for feature recognition in ground-penetrating radar (GPR) images. SVMs are capable of identifying features in GPR images with scarce data in nonlinear and high-dimensional problems [16]. Through-wall radar is similar to GPR, so we address through-wall problems using an SVM. Several studies of this method have been conducted, and the results were satisfactory. Wang and Zhang [17, 18] were able to estimate the location and the electromagnetic characteristics of the target, but the method failed to produce a clear image of the target. Other studies have performed human activity classification based on micro-Doppler signatures [19, 20], but the methods used require that the target is moving and thus do not work with a static target.

To obtain an image of a target, we first estimate the wall parameters using an SVM and then construct the image based on the estimated parameters using the BP algorithm. This paper is organized as follows. In Section 2, we discuss the imaging geometry of TWRI, and a parameter estimation approach based on SVMs for through-wall detection is proposed. In Section 3, the performance of the proposed wall parameter estimation method is assessed, and the images obtained using the BP algorithm are given. Several conclusions are discussed in Section 4.

\section{Materials and Methods}

This section presents the basic formulation for TWRI using an SVM. A simulation model is employed, and the SVM

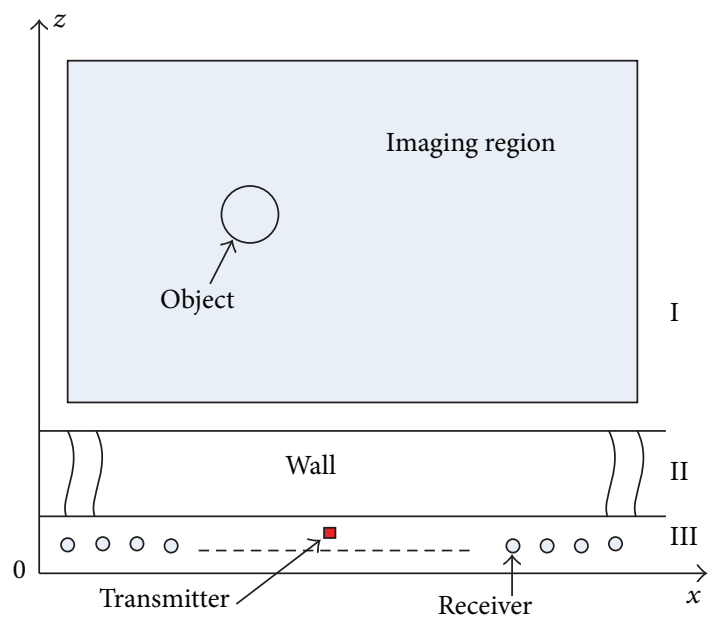

FIgURE 1: Schematic of TWRI.

is used to estimate the unknown parameters. Finally, the entropy criterion is described.

2.1. Image Reconstruction. TWRI belongs to the electromagnetic inverse problem. The information of the targets behind the walls is deduced according to limited information of receiving signals. Figure 1 shows a simple schematic of TWRI. In this study, we consider the $2 \mathrm{D}$ problem, where both the wall and the target are assumed to be infinitely high and invariant in the $y$-axis. As shown in Figure 1, the domain contains three regions: regions I and III are free space and region II is the wall. The dielectric permittivity and the magnetic permeability of the free space are denoted by $\varepsilon_{0}$ and $\mu_{0}$. The relative permittivity and the magnetic permeability of the wall are denoted by $\varepsilon_{r}$ and $\mu_{0}$, respectively, and the thickness of the wall is denoted by $d$. The transmitter position is $\left(x_{t}, z_{t}\right)$, which is $0.05 \mathrm{~m}$ from the front side of the wall. The receiver position is $\left(x_{r}, z_{r}\right)$, which is $0.04 \mathrm{~m}$ from the wall on the same side as the transmitter. The receiver moves along a scan line of length $L$ between $x_{s 1}$ and $x_{s 2}$ and collects the scattered field. The scan interval of the receiver is $0.02 \mathrm{~m}$. The target is located in the imaging region, which is behind the wall and inaccessible to the observer. The imaging region is a rectangle bounded laterally by $x_{\min }$ and $x_{\max }$ and longitudinally by $z_{\min }$ and $z_{\max }$.

Simulations were performed using the finite-difference time-domain (FDTD) method, which is an exact field computation method that has been successfully used to compute the propagation of EM waves through walls. In the FDTD simulation, the domain of the TWRI problem is discretized into square cells $1 \mathrm{~cm}$ on a side. The time resolution was $16.68 \times 10^{-12} \mathrm{~s}$. To model the EM illumination through a homogeneous wall, the transmitter signal was assumed to be an UWB short pulse, a 1.2 ns Gaussian pulse modulated by a $2 \mathrm{GHz}$ cosine wave. The receiver collects the scattered field. The scattered field at every sampling location is contained in the vector $\mathbf{E}_{\mathrm{sca}}=\left(E_{1}, E_{2}, \ldots, E_{k}\right)$, where $k$ is the index of the sampling location. The sampling location is also the receiver 
location. The vector $\mathbf{E}_{\mathrm{sca}}$ contains the only information available, and this information is limited and nonlinear in the location, the shape, the relative permittivity and conductivity of the targets and the thickness, and the relative permittivity of the wall parameters, which are all we want to know. The information of the targets can be determined directly by the scatter field based on the theory of the SVM [17, 18], but no more information can be got. So this paper is devoted to develop the relationship between the wall parameters and the scatter field. We represent the wall parameters as $Y=$ $\left(y_{i} \mid y_{i} \in C\right)$, where $C=\left(d, \varepsilon_{r}, \sigma\right)$ and $\sigma$ is the conductivity of the wall. The relationship between $\mathbf{E}_{\mathrm{sca}}$ and $Y$ is generally expressed as follows:

$$
Y=f\left(\mathbf{E}_{\mathrm{sca}}\right) \text {. }
$$

Obviously, $\mathbf{E}_{\text {sca }}$ and $Y$ have nonlinear relationship which is difficult to get directly. But in the SVM, we can suppose that $\mathbf{E}_{\mathrm{sca}}$ is the input and $Y$ is the output. Through training the input-output pairs by the SVM, the unknown function $f$ can be approximated formulated; then a simple, linear connection between $\mathbf{E}_{\mathrm{sca}}$ and $Y$ is obtained. So the uncertain wall parameters are predicted quickly and accurately from $\mathbf{E}_{\mathrm{sca}}$.

2.2. The Basic Theory of the SVM. In the SVM, the training dataset is $\left\{\left(\mathbf{E}_{\mathrm{sca} i}, y_{i}\right), i=1,2, \ldots, l\right\}$, where $\mathbf{E}_{\mathrm{sca} i} \in R^{N}$ denotes the space of the input patterns in a data vector, $y_{i} \in R$ denotes the space of the output patterns in an element, and $l$ is the number of samples. Because the receiving signal of every receiver is different under the condition of different sample, the feature can be extracted by every receiver. Thus every vector $\mathbf{E}_{\text {scai }}$ contains $N$ features, where $N$ is the number of the receivers. Since the SVM can only predict one parameter at one time, the thickness such as relative permittivity of the wall has its own mapping to the scattered data. Here we take $\mathbf{x}=\mathbf{E}_{\mathrm{sca}}, y=d$ as an example. Firstly we suppose a regression function whose form is

$$
y=f(\mathbf{x})=(\mathbf{w} \cdot \mathbf{x})+b
$$

where $(\cdot)$ denotes the dot product in $R^{N}, \mathbf{w}$ is weight vector, and $b$ is bias of the regression function. The optimization regression function based on the support vector regression (SVR) is built on structural risk minimization principle. The goal is error-free fitting all data by a linear function with proper accuracy which is $\varepsilon$. $\varepsilon$ is a constant number set in advance. At the same time the slack variables $\xi_{i}$ and $\xi_{i}^{*}$ are introduced for infeasible constraints for some acceptable errors. Therefore, the problem can be treated as an original optimization issue as follows:

$$
\begin{array}{ll}
\min _{w, b, \xi} & \left(\frac{1}{2}\|\mathbf{w}\|^{2}+\frac{C}{l} \sum_{i=1}^{l}\left(\xi_{i}+\xi_{i}^{*}\right)\right) \\
\text { s.t. } & \left(\left(\mathbf{w} \cdot \mathbf{x}_{i}\right)+b\right)-y_{i} \leq \varepsilon+\xi_{i}, \quad i=1,2, \ldots, l, \\
& y_{i}-\left(\left(\mathbf{w} \cdot \mathbf{x}_{i}\right)+b\right) \leq \varepsilon+\xi_{i}^{*}, \quad i=1,2, \ldots, l, \\
& \xi_{i}, \xi_{i}^{*} \geq 0, \quad i=1,2, \ldots, l .
\end{array}
$$

The constant $C>0$ is a penalty parameter which determines the trade-off between the flatness of $f$ and the amount up to which deviation larger than $\varepsilon$ is tolerated. The constrained optimization problem above is solved by Lagrange multiplier condition method and is transformed to its dual problem, that is,

$$
\begin{aligned}
\max _{\alpha, \alpha^{*}} & \left(\frac{1}{2} \sum_{i, j=1}^{l}\left(\alpha_{i}^{*}-\alpha_{i}\right)\left(\alpha_{j}^{*}-\alpha_{j}\right)\left(\Phi\left(\mathbf{x}_{i}\right) \cdot \Phi\left(\mathbf{x}_{j}\right)\right)+\varepsilon \sum_{i=1}^{l}\left(\alpha_{i}^{*}+\alpha_{i}\right)-\sum_{i=1}^{l} y_{i}\left(\alpha_{i}^{*}-\alpha_{i}\right)\right) \\
\text { s.t. } \quad & \sum_{i=1}^{l}\left(\alpha_{i}-\alpha_{i}^{*}\right)=0 \\
& 0 \leq \alpha_{i}, \quad \alpha_{i}^{*} \leq \frac{C}{l}, \quad i=1,2, \ldots, l .
\end{aligned}
$$

The optimal solution of the constrained quadratic programming problem (CQP) is $\bar{\alpha}=\left(\bar{\alpha}_{1}, \bar{\alpha}_{1}^{*}, \ldots, \bar{\alpha}_{l}, \bar{\alpha}_{l}^{*}\right)^{T}$, where $\alpha_{i}$ and $\alpha_{i}^{*}$ are Lagrange multipliers. $\Phi$ is a nonlinear mapping through which $\mathbf{x}_{i}$ is mapped to the higher-dimensional space $\Phi\left(\mathbf{x}_{i}\right)$. The transformed vector $\Phi\left(\mathbf{x}_{i}\right)$ and $y_{i}$ are linear in the higher-dimensional space, but $\Phi$ is unknown explicitly. To simplify the complexity of the algorithm, the kernel function $K\left(\mathbf{x}_{i}, \mathbf{x}_{j}\right)=\left(\Phi\left(\mathbf{x}_{i}\right) \cdot \Phi\left(\mathbf{x}_{j}\right)\right)$ which satisfies Mercer's condition is introduced. Frequently used kernel functions include linear function, polynomial function, radial basis function (RBF), and sigmoid function. Among the four types, the RBF has the best performance and is used in this study. The RBF is given by

$$
k\left(\mathbf{x}_{i}, \mathbf{x}_{j}\right)=\exp \left(-\gamma\left\|\mathbf{x}_{i}-\mathbf{x}_{j}\right\|^{2}\right)
$$

where $\gamma$ is the variance of the kernel function, which is determined using cross-validation. So the dot product in higher-dimensional $\left(\Phi\left(\mathbf{x}_{i}\right) \cdot \Phi\left(\mathbf{x}_{j}\right)\right)$ is solved skillfully while 


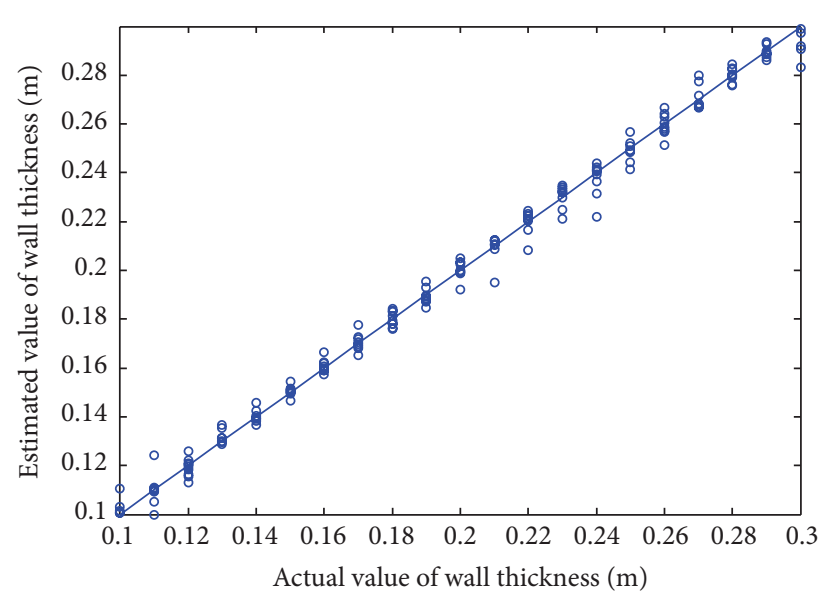

(a) Wall thickness

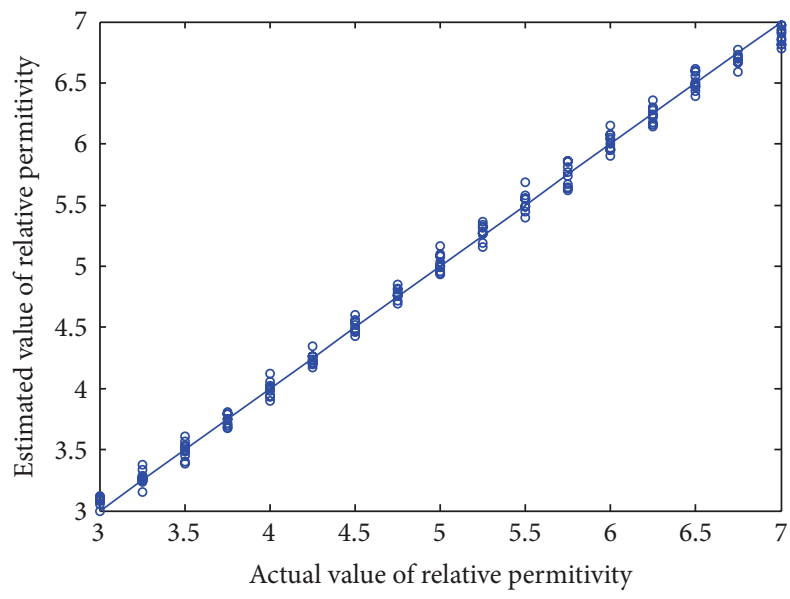

(b) Relative permittivity

FIGURE 2: Estimated and actual values of the wall parameters.

$\Phi$ is not important any longer. Then the decision function $f$ is as follows:

$$
\begin{aligned}
y & =\sum_{n=1}^{N_{\mathrm{sv}}}\left(\bar{\alpha}_{i}^{*}-\bar{\alpha}_{i}\right)\left(\Phi\left(\mathbf{x}_{n}\right) \cdot \Phi(\mathbf{x})\right)+\bar{b} \\
& =\sum_{n=1}^{N_{\mathrm{sv}}}\left(\bar{\alpha}_{i}^{*}-\bar{\alpha}_{i}\right) K\left(\mathbf{x}_{n}, \mathbf{x}\right)+\bar{b},
\end{aligned}
$$

where $\mathbf{x}_{n}$ is a training pattern whose corresponding Lagrange multiplier is nonzero, such training patterns are called support vectors (SVs) and $N_{\mathrm{sv}}$ is the number of SVs. The threshold value $\bar{b}$ which satisfies Karush-Kuhn-Tucker (KKT) condition has two expressions according to $\bar{\alpha}_{j}$ or $\bar{\alpha}_{k}^{*}$, which both in the open interval $(0, C / l)$. If $\bar{\alpha}_{j}$ is selected,

$$
\bar{b}=y_{j}-\sum_{n=1}^{N_{\mathrm{sv}}}\left(\bar{\alpha}_{i}^{*}-\bar{\alpha}_{i}\right)\left(\mathbf{x}_{n}, \mathbf{x}_{j}\right)+\varepsilon .
$$

If $\bar{\alpha}_{k}^{*}$ is selected,

$$
\bar{b}=y_{k}-\sum_{n=1}^{N_{\mathrm{sv}}}\left(\bar{\alpha}_{i}^{*}-\bar{\alpha}_{i}\right)\left(\mathbf{x}_{n}, \mathbf{x}_{k}\right)-\varepsilon .
$$

The predicted value $y$ for a new input $\mathbf{x}$ is obtained according to (6). The process of establishing the mapping for the relative permittivity is the same as the one for the thickness of the wall.

2.3. Entropy Criterion. The ultimate goal is to construct an image of the target. Using the estimated wall parameters, we can image the target behind the wall with the BP algorithm. To assess the image quality, the entropy of the image is defined as in

$$
\xi=\frac{\left(\sum \sum\left|x_{i j}\right|^{2}\right)^{2}}{\sum \sum\left|x_{i j}\right|^{4}},
$$

where $\left|x_{i j}\right|$ denotes the field amplitude of the $(i, j)$ th FDTD cell. The smaller the value of the entropy criterion is, the better the quality is. The values of the entropy criterion for the images constructed using actual and estimated values of the wall parameters are compared to test whether the image obtained with the estimated wall parameters is satisfactory.

\section{Results and Discussion}

To show the effectiveness of the method based on the SVM, results from numerical simulations are presented in this section. Referring to the schematic of TWRI shown in Figure 1 , the imaging region is a rectangle defined by $x_{\min }=0$, $x_{\max }=2.4 \mathrm{~m}, z_{\min }=0.4 \mathrm{~m}$, and $z_{\max }=3 \mathrm{~m}$. The position of the transmitter is $x_{t}=1.2 \mathrm{~m}, z_{t}=0.2 \mathrm{~m}$. The positions of the receivers are from $x_{s 1}=0.02 \mathrm{~m}$ to $x_{s 2}=2.38 \mathrm{~m}$, with $z_{r}=0.21 \mathrm{~m}$ for both. A metal target with its center located at $(0.9 \mathrm{~m}, 1.4 \mathrm{~m})$ and a radius of $0.15 \mathrm{~m}$ was assumed to exist behind the wall in the imaging region.

The training and testing data for the SVM were obtained by repeated simulations in FDTD, with variations in the wall thickness and the relative permittivity, as follows:

$$
\begin{aligned}
d & =0.1+0.01 \times m, \quad m=0,1, \ldots, 20, \\
\varepsilon_{r} & =3+0.25 \times n, \quad n=0,1, \ldots, 16 .
\end{aligned}
$$

One-half of the dataset was used for the training dataset, and the other half of the dataset was used as the testing dataset. The model was obtained after the training of the SVM and then was applied to the testing dataset to get the estimation results. Figure 2 displays the relationships between the actual and estimated values of the wall parameters, that is, the thickness and the relative permittivity of the wall.

Figure 3 shows the errors in the estimation of the two wall parameters. In Figure 3(a), the estimation error for the wall thickness is generally less than $0.005 \mathrm{~m}$. In Figure 3(b), the estimation error for the relative permittivity is generally less 


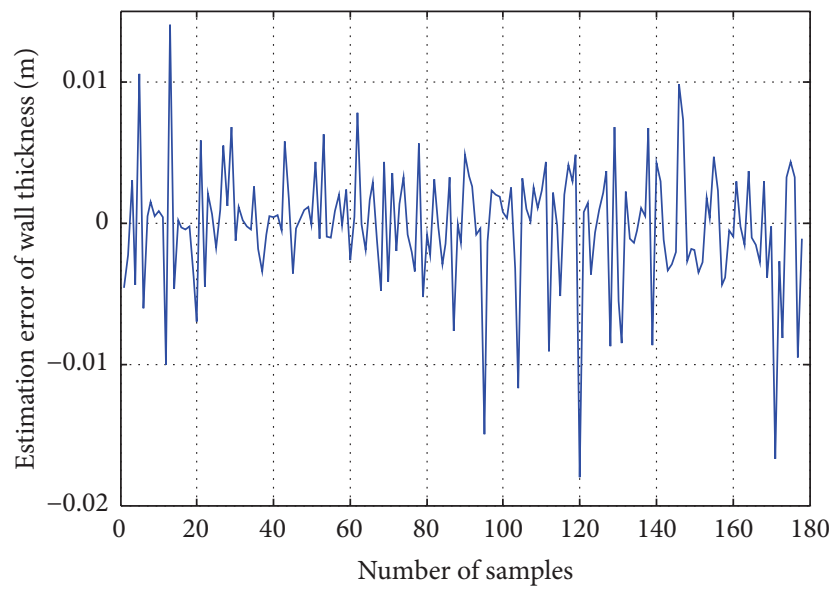

(a) Wall thickness

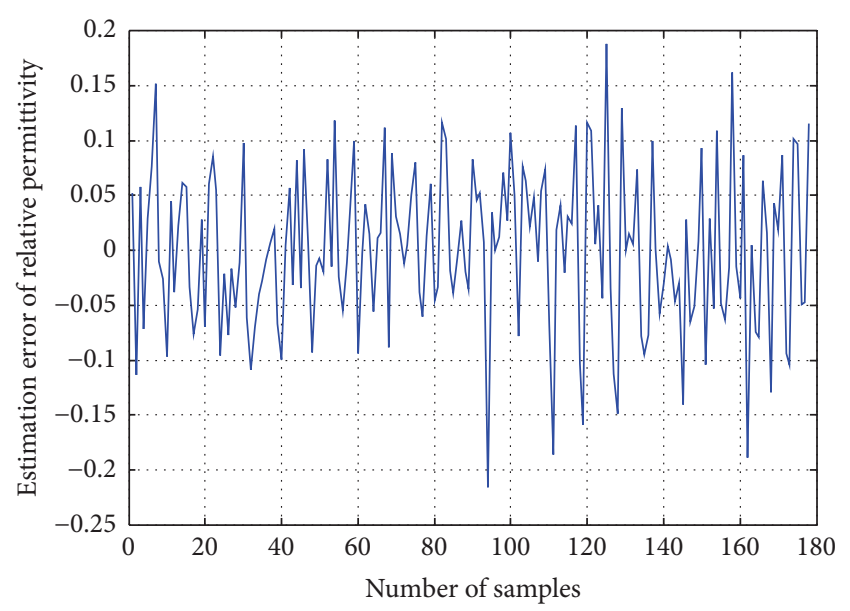

(b) Relative permittivity

FIGURE 3: The estimation errors for the wall parameters.

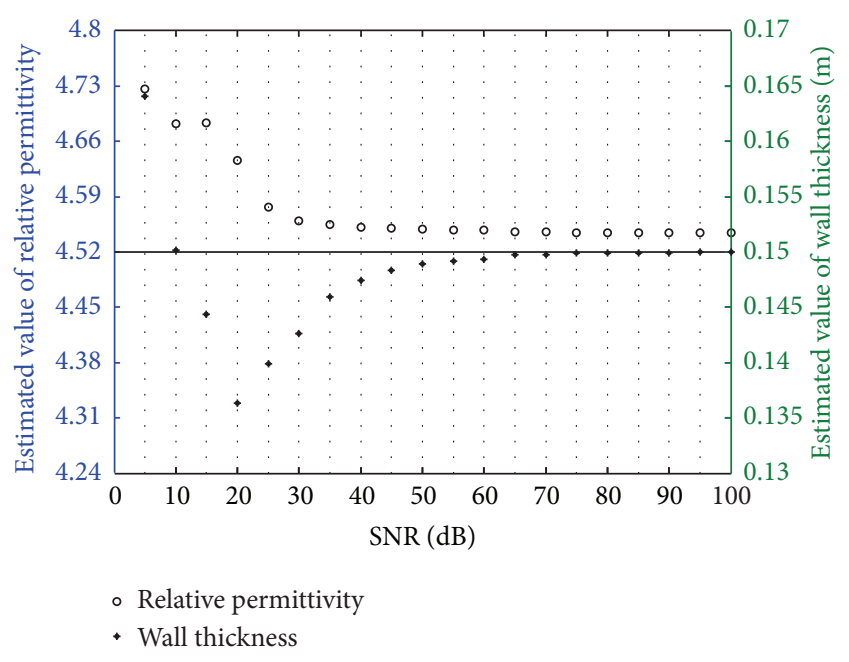

FIGURE 4: Estimated values of the wall parameters versus SNR.

than 0.1. So the SVM can estimate the wall parameters with small errors in TWRI. Moreover, if the model is obtained, the estimation time is no more than $1 \mathrm{~s}$. The estimation approach based on the SVM will not increase the imaging time.

The above-mentioned analysis is for noiseless circumstance. In reality, it is important that the estimated method is robust under noise contamination. So we also simulate the noisy environment. In FDTD simulation a wall is added with the actual values of $\varepsilon_{r}=4.52, d=0.15 \mathrm{~m}$. Other parameters are the same as before, except that $5,10, \ldots, 100 \mathrm{~dB}$ Gaussian noise whose interval is $5 \mathrm{~dB}$ is added in $\mathbf{E}_{\mathrm{sca}}$, and the results are shown in Figures 4 and 5. In Figure 4, the estimated value of relative permittivity and wall thickness is given and the horizontal line represents their actual value. The estimated value of relative permittivity is always higher than the actual value and decreases gradually with the increasing of the noise. The changing trend of the wall thickness is approximately the same as the relative permittivity. The estimated value and the actual value of wall thickness are almost identical when SNR is higher than $65 \mathrm{~dB}$.

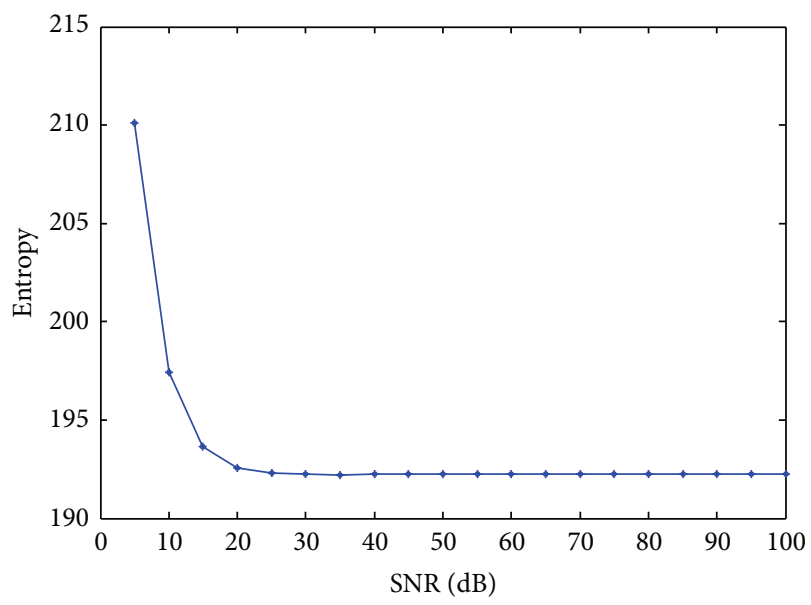

FIGURE 5: Entropy versus SNR.

Figure 5 displays the entropy under noisy situation. The entropy keeps almost unchanged when SNR is higher than $25 \mathrm{~dB}$. Figure 5 combined with Figure 4 shows that the highquality imaging is got when the error is concealed out or the predicted value is almost equal to the actual value $[12,15]$ when SNR is no less than $25 \mathrm{~dB}$.

We note that, in this example, the target is known, and we can estimate the wall parameters very well and detect the target successfully. However, in many applications, the target is unknown, as are the wall parameters. This is a challenge for the method. As previously discussed, the target is a cylinder. With the center of the target unchanged, the wall parameters for other values of the target radius were estimated. We selected the values $r=0.05 \mathrm{~m}, 0.07 \mathrm{~m}, \ldots, 0.25 \mathrm{~m}$ in increments of $0.02 \mathrm{~m}$. Figure 6 shows the estimated values of the wall parameters for the various values of the target radius. The estimated values are not equal to the actual ones, and several are quite different. We observe that if $r<0.15 \mathrm{~m}$, the estimated value of the wall thickness is greater than the actual value and the estimated value of the relative permittivity is 


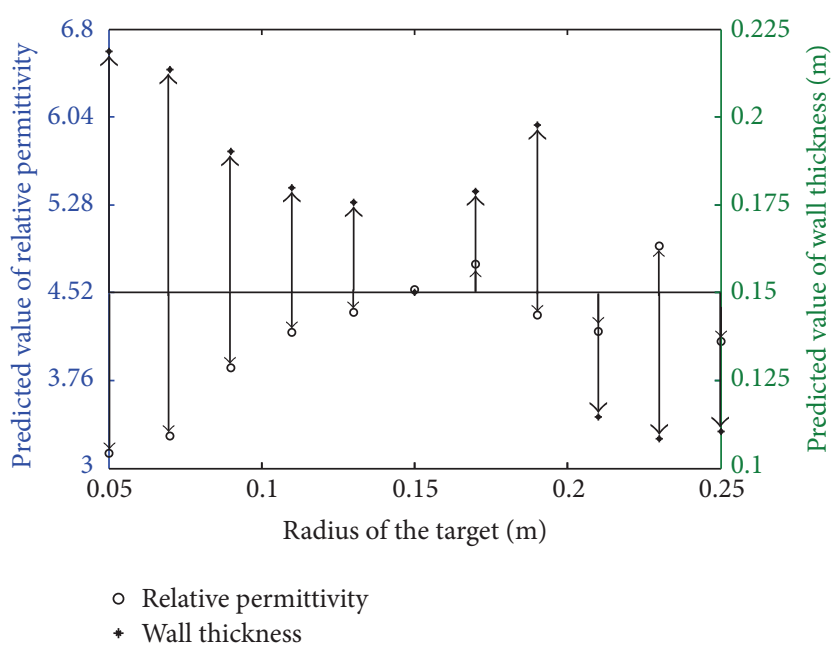

Figure 6: The estimated values of the wall parameters versus radius of the target.

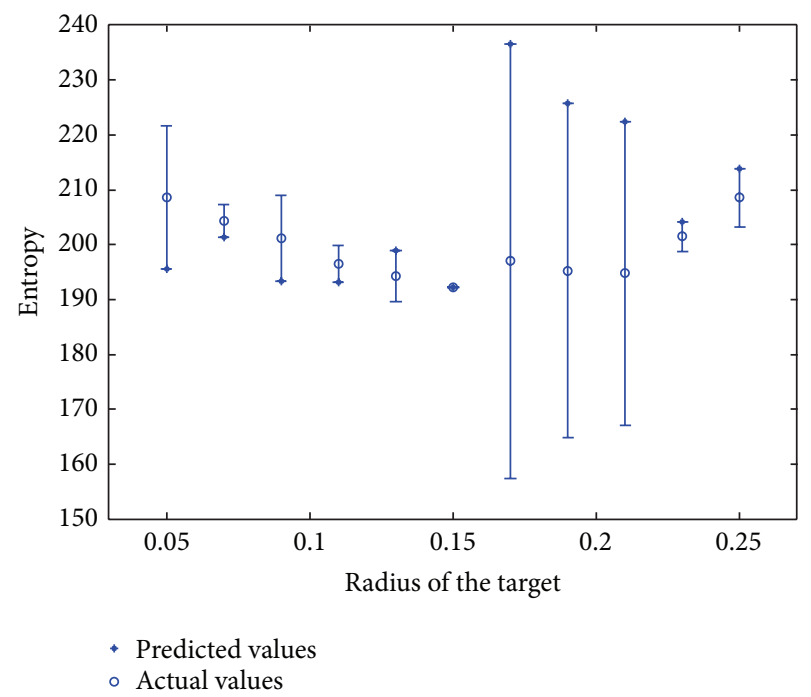

FIGURE 7: The errorbar of the entropy of the images with the actual and estimated wall parameters.

less than the actual value. However, if $r>0.15 \mathrm{~m}$, this trend is not followed.

From Figure 7 we know that the entropy of the image reaches the minimum when $r=0.15 \mathrm{~m}$ no matter with the actual values of the wall parameters or the estimated ones, and the error is nearly zero. This is because the model was constructed for $r=0.15 \mathrm{~m}$. At this radius, the estimated values of the wall parameters are the most consistent with the actual ones, so the image quality is the best.

When $r \neq 0.15 \mathrm{~m}$, the entropy for the estimated values of the wall parameters differs from that for the actual values. However, although the values of the wall parameters are not correctly estimated, a reasonable value for the entropy nevertheless can be achieved. In Figure 7, the entropy with the estimated wall parameters is greatest for $r=0.17 \mathrm{~m}$. Because the estimated values of the wall parameters are both higher than the actual ones, the image quality is the worst.

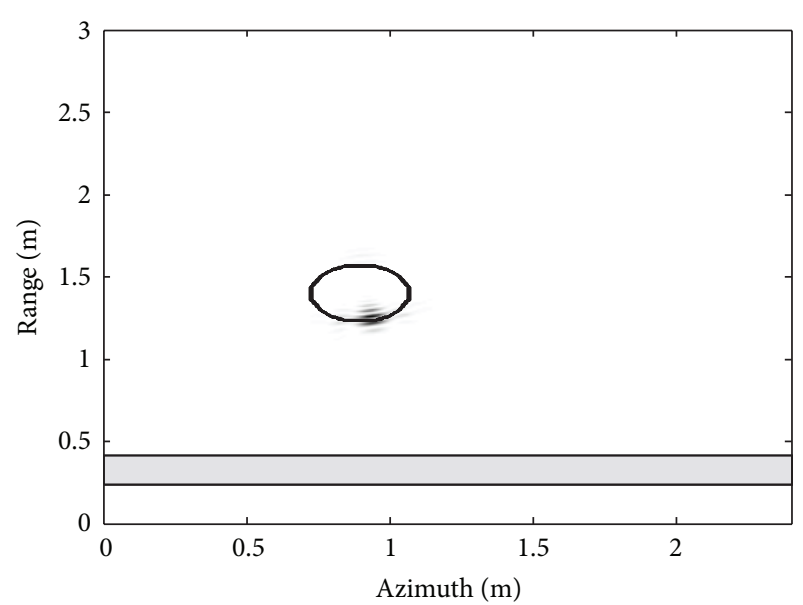

(a) Estimated values with $\mathrm{SNR}=5 \mathrm{~dB}$

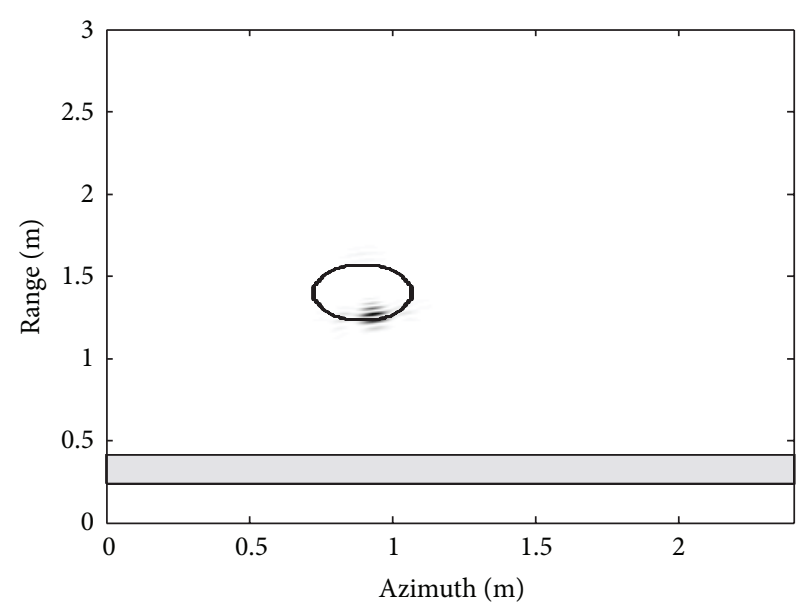

(b) Estimated values with $\mathrm{SNR}=35 \mathrm{~dB}$

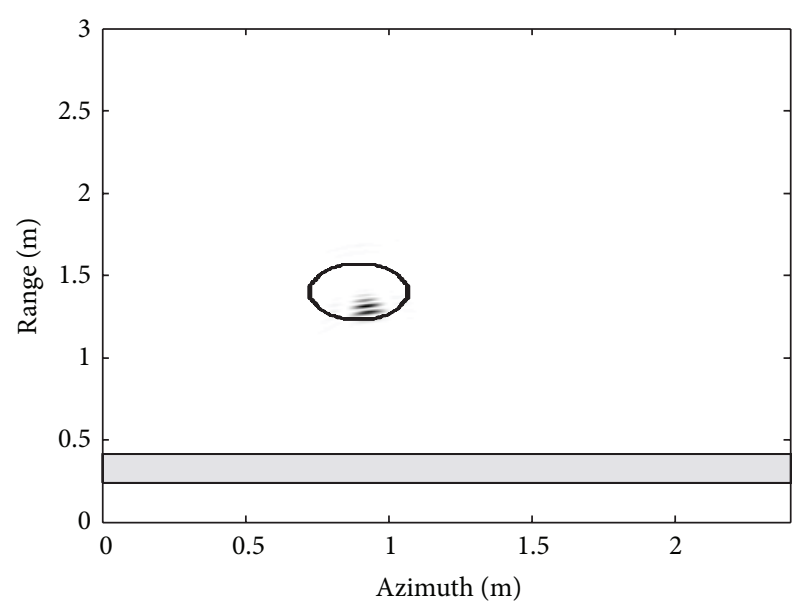

(c) Actual value

FIGURE 8: BP images with $r=0.17 \mathrm{~m}$ according to wall parameters.

To check the robust of the approach, Figures $8(\mathrm{a}), 8(\mathrm{~b})$, and $8(\mathrm{c})$ give the BP image results with the target radius of $0.17 \mathrm{~m}$ in estimated wall parameters with $5 \mathrm{~dB}$ noise, 
estimated value with $35 \mathrm{~dB}$ noise, and actual values. The estimated wall parameters with $5 \mathrm{~dB}, 35 \mathrm{~dB}$ are $\varepsilon_{r 5}=4.7259$, $d_{5}=0.164 \mathrm{~m}, \varepsilon_{r 35}=4.5546, d_{35}=0.146 \mathrm{~m}$, respectively. In all cases, the target image is well focused, but the location of the target image constructed with the estimated wall parameters is closer to the wall (i.e., in the range direction) than that of the target image constructed with the actual wall parameters. The imaging quality with noise is slightly inferior to that with actual values.

\section{Conclusions}

Knowledge of the exact values of the wall parameters permits the construction of focused images and the determination of the exact position of an object behind a wall. Unfortunately, the wall characteristics such as the thickness and the relative permittivity are unknown parameters in practical TWRI applications. In this study, a novel approach based on SVMs is used to estimate the values of the wall parameters. The approach can estimate the wall thickness and the relative permittivity quickly and accurately. Images obtained using the BP algorithm validate the efficiency of the proposed method. In a realistic situation, the estimated values of the wall parameters can be used to form a reasonably focused image regardless of the estimation error. The approach is robust and high-fidelity under noisy situation. In this study, the target behind the wall is cylindrical in shape; other shapes are also suitable. In addition, the approach based on SVMs is shown to be effective for the case of a single target, but this approach is not suitable for the case of multiple targets. Estimation of the wall parameters when there are multiple targets will be studied in the future.

\section{Conflict of Interests}

The authors declare that there is no conflict of interests regarding the publication of this paper.

\section{Acknowledgment}

This work is supported by the National Natural Science Foundation of China (Grant nos. 61071022 and 61372045).

\section{References}

[1] L. J. Kong, G. L. Cui, X. B. Yang, and J. Y. Yang, "Three-dimensional human imaging for through-the-wall radar," in Proceedings of the IEEE Radar Conference, pp. 1-4, IEEE, Pasadena, Calif, USA, May 2009.

[2] F. Ahmad, M. G. Amin, and S. A. Kassam, "Synthetic aperture beamformer for imaging through a dielectric wall," IEEE Transactions on Aerospace and Electronic Systems, vol. 41, no. 1, pp. 271-283, 2005.

[3] F. Ahmad, Y. Zhang, and M. G. Amin, "Three-dimensional wideband beamforming for imaging through a single wall," IEEE Geoscience and Remote Sensing Letters, vol. 5, no. 2, pp. 176-179, 2008.
[4] L. Li, W. Zhang, and F. Li, "A novel autofocusing approach for real-time through-wall imaging under unknown wall characteristics," IEEE Transactions on Geoscience and Remote Sensing, vol. 48, no. 1, pp. 423-431, 2010.

[5] W. J. Zhang, L. L. Li, and F. Li, "Autofocusing imaging through the unknown building walls," in Proceedings of the Asia Pacific Microwave Conference (APMC '08), pp. 1-5, December 2008.

[6] G. Y. Wang and M. G. Amin, "Imaging through unknown walls using different standoff distances," IEEE Transactions on Signal Processing, vol. 54, no. 10, pp. 4015-4025, 2006.

[7] G. Y. Wang, M. G. Amin, and Y. Zhang, "New approach for target locations in the presence of wall ambiguities," IEEE Transactions on Aerospace and Electronic Systems, vol. 42, no. 1, pp. 301-315, 2006.

[8] P. Protiva, J. Mrkvica, and J. MacHáč, "Estimation of wall parameters from time-delay-only through-wall radar measurements," IEEE Transactions on Antennas and Propagation, vol. 59, no. 11, pp. 4268-4278, 2011.

[9] P. Protiva, J. Mrkvica, M. Safarik, R. Sikl, V. Vlasak, and J. Machac, "An improved technique for estimating wall parameters from time-delay through-wall radar measurements," in Proceedings of the 22nd International Conference on Radioelektronika, pp. 1-4, April 2012.

[10] S. Kaushal and D. Singh, "Role of signal processing for estimating the wall thickness for TWI system," in Proceedings of the 4th International Conference on Computing, Communications and Networking Technologies (ICCCNT '13), pp. 1-7, IEEE, Tiruchengode, India, July 2013.

[11] Q. Chai and Y. Li, "Parameters estimation by multi-channel measuring for through-wall target positioning," in Proceedings of the 2nd Annual Conference on Electrical and Control Engineering (ICECE '11), pp. 742-745, IEEE, Yichang, China, September 2011.

[12] Y. Jia, L. J. Kong, X. B. Yang, and K. D. Wang, "A new approach for wall parameters estimation in through-the-wall radar imaging," in Proceedings of the Radar Conference (RADAR '11), pp. 95-98, IEEE, Kansas City, Mo, USA, May 2011.

[13] Z. X. Zhao, L. J. Kong, and J. G. Liu, "An estimation approach for equivalent wall parameters in through-wall-radar imaging," in Proceedings of the IET International Radar Conference, pp. 1-4, Xian, China, April 2013.

[14] X. Gu and Y. H. Zhang, "Autofocus imaging simulation for through-wall radar by using FDTD with unknown wall characteristics," in Proceedings of the Asia-Pacific Microwave Conference (APMC '10), pp. 1657-1660, December 2010.

[15] T. Jin, B. Chen, and Z. M. Zhou, "Image-domain estimation of wall parameters for autofocusing of through-the-wall SAR imagery," IEEE Transactions on Geoscience and Remote Sensing, vol. 51, no. 3, pp. 1836-1843, 2013.

[16] X. Y. Xie, P. Li, and L. B. Liu, "GPR identification of voids inside concrete based on support vector machine (SVM) algorithm," in Proceedings of the 14th International Conference on Ground Penetrating Radar (GPR '12), pp. 381-386, IEEE, Shanghai, China, June 2012.

[17] F. F. Wang and Y. R. Zhang, "The support vector machine for dielectric target detection through a wall," Journal of Electromagnetic Waves and Applications, vol. 25, pp. 75-84, 2011.

[18] F.-F. Wang and Y.-R. Zhang, "An electromagnetic inverse scattering approach based on support vector machine," Acta Physica Sinica, vol. 61, no. 8, pp. 084101-1-084101-7, 2012 (Chinese). 
[19] Y. Kim and H. Ling, "Through-wall human tracking with multiple doppler sensors using an artificial neural network," IEEE Transactions on Antennas and Propagation, vol. 57, no. 7, pp. 2116-2122, 2009.

[20] X. X. Liu, H. Leung, and G. A. Lampropoulous, "An intelligent through-the-wall recognition system for homeland security," in Proceedings of the IEEE World Congress on Computational Intelligence, pp. 2084-2090, Hong Kong, 2008. 

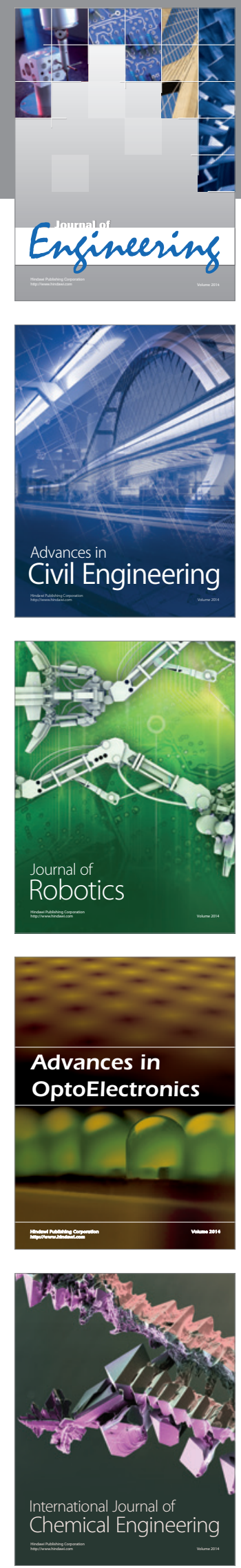

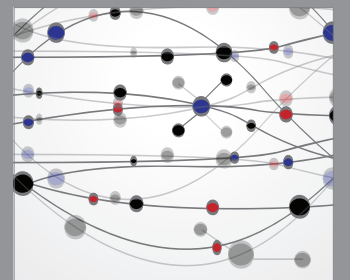

The Scientific World Journal
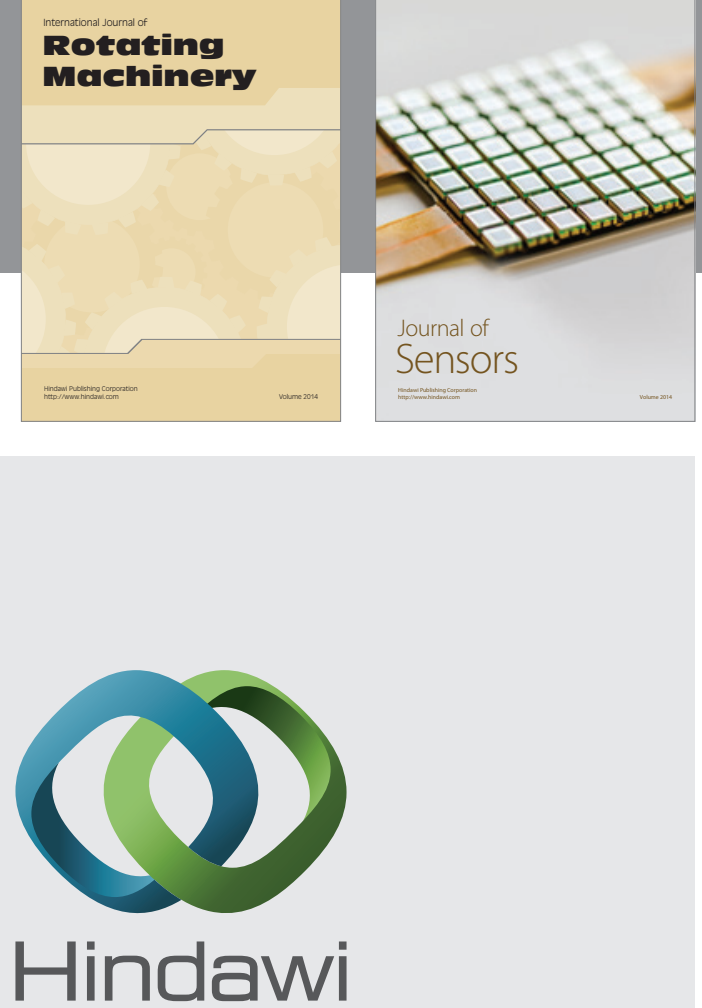

Submit your manuscripts at http://www.hindawi.com
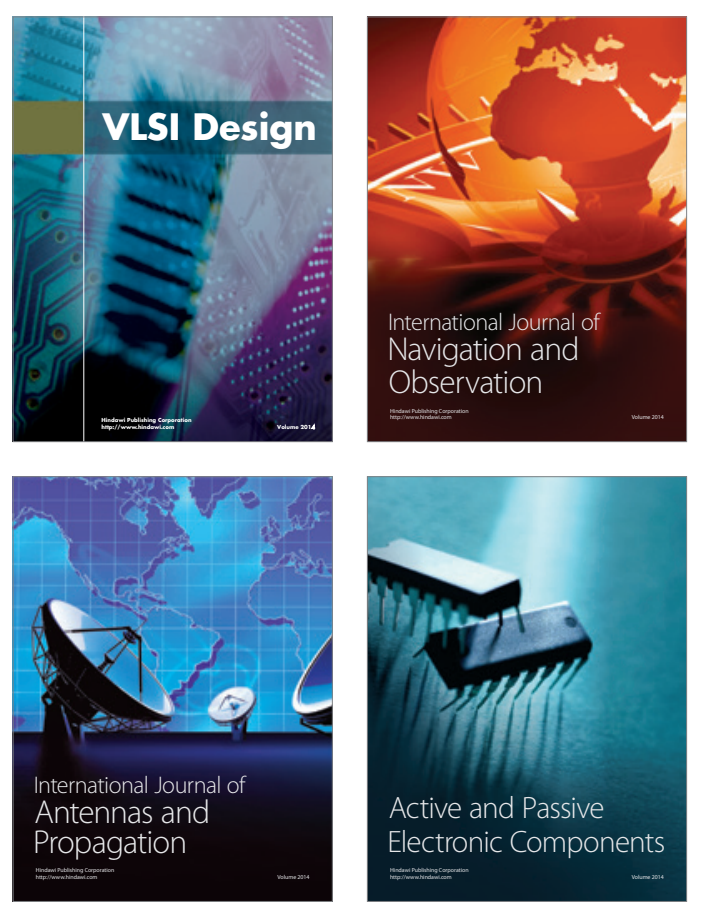
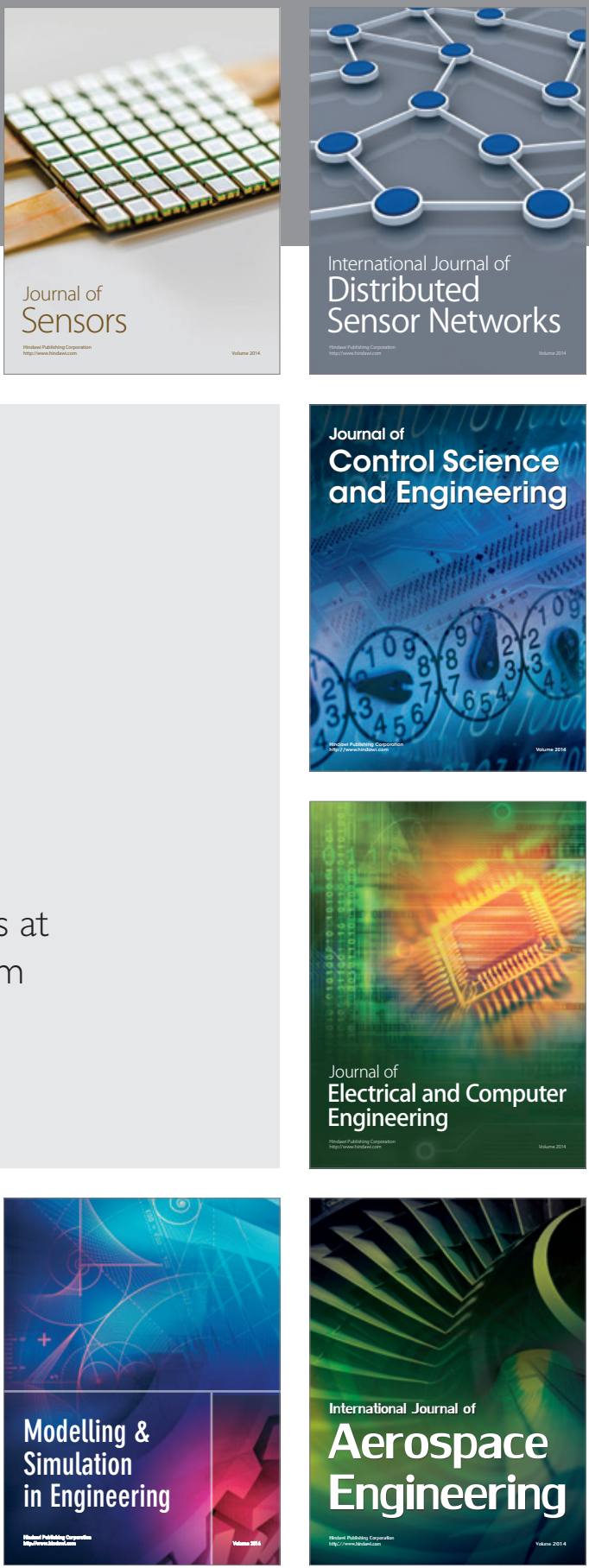

Journal of

Control Science

and Engineering
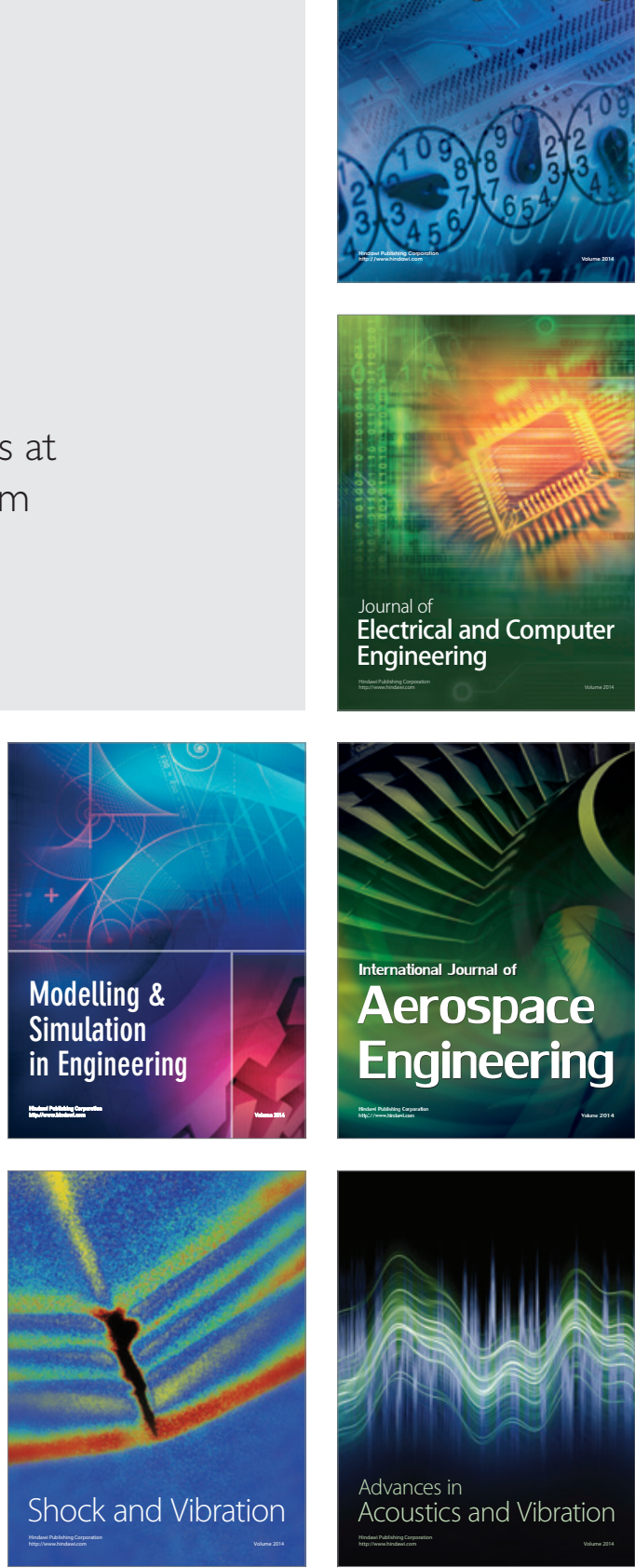